\title{
James B. Conant y Thomas S. Kuhn. Líneas de continuidad en el estudio histórico de la ciencia*
}

\author{
James B. Conant and Thomas S. Kuhn. \\ Lines of continuity in the historical study of science
}

Pablo Antonio Pacheco

\begin{abstract}
Resumen
El presente trabajo delimita líneas comunes a los enfoques de los historiadores de la ciencia James Conant y Thomas Kuhn. Exceptuando aquellas características y particularidades de sus pensamientos acerca del desarrollo histórico de la ciencia, pueden identificarse aspectos que expresan intereses y preocupaciones comunes.

El recurso a la historia de la ciencia, la comparación entre la práctica científica y el desarrollo psicogenético de los niños, el análisis de los factores institucionales que inciden sobre la ciencia, la comprensión del proceso histórico de la ciencia a través de determinados esquemas conceptuales, la caracterización de una dialéctica particular entre hechos y teorías, la consideración del factor técnico en el desarrollo científico, así como la crítica de la función pedagógica de los libros de texto en la formación de los jóvenes investigadores, constituyen líneas de continuidad entre el pensamiento de ambos.
\end{abstract}

Palabras clave: esquemas conceptuales - estudios de casos - Kuhn - historia de la ciencia

\begin{abstract}
This paper defines common lines to the approaches of the historians of science James Conant and Thomas Kuhn. Excluding those special characteristics of their thought about the historical development of science, aspects that express common interests and concerns may be identified.

The appeal to the history of science, the comparison between the practice of science and psychogenetic development of children, the analysis of the institutional factors that affect science, the understanding of the historical process of science through certain conceptual schemes, the characterization of a particular dialectic between facts and theories, the consideration of technical factors in scientific development as well as the criticism of the educational function of textbooks in the training of young researchers, constitute lines of continuity between both authors.

Keywords: conceptual schemes - case studies - Kuhn - history of science

* Recibido: 20 Febrero 2011. Aceptado en versión revisada: 11 septiembre 2011.

† Secretaría de Ciencia, Técnica y Posgrado, Facultad de Filosofía y Letras, Universidad Nacional de Cuyo. Para contactar al autor, por favor escriba a: pablopach@hotmail.com.

$\$$ El presente artículo ha sido elaborado en el marco del proyecto dirigido por el profesor Guillermo A. Cuadrado y financiado por la Secretaría de Ciencia, Técnica y Posgrado de la Universidad Nacional de Cuyo, Mendoza, Argentina. El autor agradece al doctor Juan Manuel Torres por sus comentarios y a los evaluadores por sus cordiales críticas, que han permitido mejorar el manuscrito.

Metatheoria 2(1) (2011): 3-21. ISSN 1853-2322.

(C) Editorial de la Universidad Nacional de Tres de Febrero. Publicado en la República Argentina.
\end{abstract}




\section{Introducción}

La mayor parte de los trabajos que analizan la génesis del pensamiento de Thomas Kuhn se centran en sus vinculaciones con los planteos de Stanley Cavell, Robert Merton, Michael Polanyi, Willard Quine, Ludwik Fleck, Alexandre Koyré, Ludwig Wittgenstein, Karl Popper, Imre Lakatos o Paul Feyerabend, entre otros. Menos explorada ha sido la incidencia de otros como Boris Hessen, Norwood Russell Hanson, Stephen Toulmin, Bernard Cohen, Pierre Duhem, Jean Piaget o su propio mentor, el químico James Conant.

Este trabajo se propone abordar la influencia de algunos planteos de este último en la génesis de ideas claves del pensamiento de Kuhn, considerando algunos puntos que evidencian cierta continuidad en la reflexión de ambos.

La relación personal y profesional entre James Bryant Conant (1893-1978) y Thomas Samuel Kuhn (1922-1996) en el marco de la Universidad de Harvard es conocida y ha sido puesta de relieve por ellos en varias oportunidades. Sobre su vinculación con James Conant, Kuhn expresa en la entrevista autobiográfica realizada por Baltas, Hindi y Gavroglu: "Yo fui una de las dos personas a las que Conant pidió que le ayudaran. La primera vez dio el curso basado en el librito On Understanding Science, que había sido el resultado de las Conferencias Terry en Yale. Acepté entusiasmado; y nunca he olvidado la ocasión en que le conocî" (Kuhn 2000, v.i., p. 275; v.c., p. 322).

Conant obtiene el grado de doctor en filosofía en 1916 y un año más tarde trabaja como instructor y luego como profesor asistente y en ejercicio en Harvard, convirtiéndose en Presidente de esta en 1933. En este período sus preocupaciones se centran en la reforma de la enseñanza en los Estados Unidos, impulsando especializaciones como el grado de Maestría en Artes de la Enseñanza y colaborando como asesor en la Comisión Nacional de Educación de su país. Durante la Segunda Guerra Mundial participa como consultor de la Fundación Nacional de Ciencia y de la Comisión de Energía Atómica. Asimismo, interviene en proyectos vinculados a desarrollos militares. Luego de la guerra Conant deja su administración en la universidad y en 1953 es nombrado por el gobierno de Estados Unidos Embajador en Alemania Occidental. ${ }^{1}$

Kuhn, por su parte, se gradúa como bachiller en física en 1943, obtiene su maestría en 1946 y su doctorado en 1949 en la Universidad de Harvard. Enseña historia y filosofía de la ciencia en dicha universidad (1951-1956), en la Universidad de California (1956-1964), en Princeton (1964-1979) y en el Instituto Tecnológico de Massachusets (1979-1991), donde fue nombrado profesor emérito.

Ambos historiadores se conocieron en Harvard a fines de la década de 1940, cuando Conant era presidente y Kuhn estudiante de física. La influencia de las ideas de Conant sobre el pensamiento y los trabajos de Kuhn es en cierta forma decisiva, según lo admite este último cuando afirma:

\footnotetext{
${ }^{1}$ Para ampliar los aspectos biográficos de Conant, véase Hershberg (1993).
} 
Muchos han sido los amigos y colegas que con sus valiosas advertencias y críticas han colaborado en la creación de este libro, pero ninguno de ellos dejó tan profunda huella como el embajador James B. Conant. Fue él quien me persuadió, mientras trabajaba a su lado, de que el estudio de la historia de la ciencia podía proporcionar un nuevo tipo de comprensión acerca de la estructura y función de la investigación científica. Sin mi propia revolución copernicana, que Conant engendró, el presente libro y mis otros ensayos sobre historia de la ciencia nunca habrían sido escritos. (Kuhn 1957, v.i., p. ix; v.c., p. 12)

En el mismo sentido, al publicar en 1962 su ensayo más importante, La estructura de las revoluciones científicas, expresa:

Quien por vez primera me introdujo en la historia de la ciencia fue James B. Conant, a la sazón rector de la Universidad de Harvard, con lo que inició la transformación de mi concepción acerca de la naturaleza del avance de la ciencia. Desde que comenzó dicho proceso, se ha mostrado generoso con sus ideas, con sus críticas y con su tiempo, incluyendo el tiempo preciso para leer y sugerir importantes cambios en el borrador del manuscrito. (Kuhn 1970, v.i., p. xi; v.c., p. 54)

Steve Fuller (2001) ha señalado la existencia de similitudes entre el libro La ciencia y el sentido común de Conant y La estructura de las revoluciones científicas de Kuhn, aunque no las desarrolla ni profundiza. Este vínculo entre Conant y Kuhn enmarca la génesis de algunos aspectos compartidos por el pensamiento de ambos, que se resumen en siete puntos de contacto abordados en cada uno de los siguientes apartados.

\section{Los estudios de casos y la explicación histórica de los métodos científicos}

Un primer rasgo que comparten Conant y Kuhn es el recurso a un modo de clarificación y explicación de los métodos, técnicas y procedimientos científicos, a partir del material que les brinda la aproximación de una metodología histórica.

La necesidad de encontrar adecuados métodos para elucidar y reconstruir las posibilidades, los límites y el impacto histórico del conocimiento científico y los desarrollos tecnológicos se convierte en una exigencia del sentido común generada por el contexto de posguerra a partir de la segunda mitad del siglo XX. ${ }^{2}$ En tal sentido, en La comprensión de la ciencia, publicado en 1947 y reimpreso en 1951, Conant distingue dos caminos para analizar la actividad científica, cuando sostiene:

Pues hay dos modos de investigar las complejas actividades humanas y sus productos: uno es volver a recorrer los pasos que han dado lugar a determinados resultados, y otro hacer la disección del resultado, con la esperanza de poner al descubierto su tipo estructural y revelar las relaciones lógicas de sus partes

\footnotetext{
2 "Desde el final de la Segunda Guerra Mundial no ha ocurrido nada que reduzca la importancia de que el profano entienda al hombre de ciencia. Todo lo contrario. Ha habido un creciente interés por parte del público en general en torno de los métodos de las ciencias naturales" (Conant 1947, v. c., p. 9).
} 
componentes e, incidentalmente, de exponer también sus inconsistencias y fallas. Las mentes matemáticas y filosóficas prefieren el enfoque lógico, pero yo creo que para nueve de cada diez personas el método histórico dará lugar a una comprensión más real de un tema complejo (Conant 1947, v. c., p. 28).

Se decide por un método que ofrece la aproximación histórica al estudio de la ciencia y que permite recorrer y reconstruir los pasos dados por los investigadores para obtener sus resultados. De este modo, será el primero en incorporar el llamado "método de casos" como herramienta pedagógica para esclarecer la actividad científica a la mirada del sentido común.

El "método de casos" fue diseñado y sistematizado en 1870 por el jurista norteamericano y decano de Harvard Christopher Columbus Langdell, en el marco de la enseñanza del derecho y en contraposición a los métodos pedagógicos tradicionales. Estos ponían el acento en un aprendizaje memorístico apoyado por densos tratados (método Dwight). El "método de casos" o "sistema de casos" -como lo denominó Langdell- fue posteriormente aceptado como forma de enseñanza en la escuela de negocios de la misma universidad con el nombre de "método de problemas" (problems method), lo que orientó la enseñanza hacia la resolución de problemas.

Conant piensa en la propuesta pedagógica de Langdell como una de las invenciones más importantes del siglo XIX en Estados Unidos (Conant 1965, pp. 43-45). ${ }^{3}$ Asimismo, afirma que es una herramienta didáctica efectiva para orientar el estudio histórico de la ciencia por quienes no están familiarizados con los métodos científicos. Sobre esto expresa: "Ya que sostengo que el profano puede comprender mejor la ciencia mediante el estudio atento de unos cuantos casos históricos relativamente sencillos, no me queda más alternativa que presentar algunos fragmentos de historia científica” y, más adelante, agrega: "También extraigo confianza del conocimiento de la forma en que el método de los casos en escuelas de Derecho, y un método algo similar en la Escuela Comercial de Harvard han demostrado el valor de este tipo de recurso pedagógico" (Conant 1947, v.c., pp. 17 y 32).

Para él, el método de casos recupera la tradición inductiva-empírica que impregna el conocimiento de sentido común de los no científicos, para ofrecer una mejor aproximación a la actividad científica (Conant 1965, pp. 81-82). De este modo, Conant propone abordar el estudio y la enseñanza histórica de la ciencia a través del método de casos, con el objetivo de facilitar una adecuada comprensión del desarrollo científico. Para ello, emprende la tarea de diseñar el material necesario para tal fin, como es la elaboración -bajo su dirección y en conjunto con Leonard Nash y otros colegas- de una serie de casos históricos de la ciencia publicados en 1957 bajo el título Harvard Case Histories in Experimen-

\footnotetext{
${ }^{3}$ Sobre Langdell y su invención afirma Conant: "Un aspecto altamente significativo del impacto de la nueva ciencia sobre la educación americana después de la guerra civil, fue el desarrollo del método de caso de estudio de la ley, por el nuevo Decano de la Escuela de Ley de Harvard, Christopher Columbus Langdell". Y más adelante agrega: "Para mí, por lo tanto, Langdell puede ser ubicado entre los grandes inventores americanos del siglo diecinueve" (Conant 1965, pp. 43 y 45; traducción mía).
} 
tal Science (Conant 1957a). ${ }^{4}$ En relación con el enfoque histórico presentado en esta selección de casos, afirma:

Varias son las propuestas lanzadas y diversos los experimentos puestos en marcha para encontrar nuevos tipos de cursos científicos que puedan formar parte de un programa general de educación o de un programa de humanidades. En particular, se ha recomendado un mayor énfasis en el estudio de la historia de la ciencia, propuesta a la que me he adherido con entusiasmo. En el momento presente, la experiencia efectuada en el College de Harvard sobre un determinado tipo de enfoque histórico ha incrementado mi convicción en las posibilidades inherentes al estudio de la historia de la ciencia, particularmente si se combina con un análisis de los varios métodos por los que esta ha progresado. Aun reconociendo el valor educativo de una visión de conjunto sobre la historia de la ciencia en los últimos 300 años, creo que puede obtenerse un mayor beneficio a través de un estudio intensivo de ciertos episodios particulares en el desarrollo de la física, la química o la biología. Esta convicción se ha materializado a través de una serie de folletos titulada "Harvard Case Histories in Experimental Science". (Conant 1957b, v.i., pp. xvi-xvii; v.c., p. 19)

$\mathrm{Al}$ referirse a la finalidad de este material elaborado por él y sus colaboradores, Conant sostiene:

Los "Harvard Case Histories in Experimental Science" fueron diseñados ante todo para estudiantes especializados en las humanidades o las ciencias sociales. Dichos estudiantes requieren un entendimiento de la ciencia que les ayudará a relacionar los desarrollos de las ciencias naturales con los de los otros campos de la actividad humana. Para hacer esto, es necesaria una comprensión tanto de los métodos de la ciencia experimental como del crecimiento de las investigaciones científicas como actividad organizada de la sociedad (Conant 1957 a, p. vii). ${ }^{5}$

En otra parte, precisa que "El objetivo de los mismos ha sido desarrollar en el estudiante una cierta comprensión de la interrelación entre teoría y experimento y de la complicada cadena de razonamientos que conecta la verificación de una hipótesis con los resultados experimentales obtenidos" (Conant 1957b, v.i., p. xvii; v.c., p. 19). Es decir, interesa clarificar en cada situación, en cada episodio, la dinámica que se ha planteado históricamente entre teoría y hechos.

En un sentido similar, la elección del método histórico implica para Kuhn no solo una forma más adecuada para el estudio de las ciencias, sino un modo de acercamiento entre la práctica científica real y los filósofos de la ciencia, cuando sostiene que:

la historia de la ciencia puede contribuir a salvar la brecha que hay entre filósofos de la ciencia y la propia ciencia, la cual puede ser para ellos tanto una fuente de problemas como de datos. [...] la historia brinda, de entre varios

\footnotetext{
${ }^{4}$ Fuller (2001, p. 183) hace referencia a la utilización del método de estudio de caso en los cursos y materiales elaborados por Conant, Nash y Kuhn, aunque sin mencionar a Langdell. También Hershberg (1993, pp. 409-410) menciona estas experiencias en Harvard. Para un análisis más detallado de la incorporación de los estudios de casos como metodología de la historia de la ciencia, véase Pacheco (2005).

${ }^{5}$ La traducción es mía. Un fragmento de esta cita puede encontrarse en Solís (2007, p. 26).
} 
métodos posibles, el más práctico y accesible, gracias al cual el filósofo podría familiarizarse con la ciencia. (Kuhn 1977, v.i., p. 13; v.c., pp. 37-38)

El historiador reconoce que fue por iniciativa de Conant que se incorporó al estudio de caso como forma de comprender el desarrollo histórico de la ciencia, cuando expresa:

\begin{abstract}
Aquí estaba yo: no había terminado mi tesis de física, era insensible a ese tipo de temas - para entonces había leído las pruebas de imprenta de On Understanding Science- y ime pedían que preparara, para ese curso, un estudio de caso tomado de la historia de la mecánica? iCaramba! Pero Conant era así, hacía ese tipo de cosas [...]. Creo que Conant nunca vio, en la misma medida que yo, ni en los casos históricos que preparaba, ni en sus clases, la importancia de explicar las creencias que la gente había tenido antes. (Kuhn 2000, v.i., p. 275; v.c., p. 322) ${ }^{6}$
\end{abstract}

Para Kuhn tanto como para Conant, ${ }^{7}$ el método histórico constituye una aproximación adecuada para comprender las teorías o conceptos científicos y sus vinculaciones con los hechos por medio del análisis de casos concretos. En su trabajo de 1957, el primero afirma:

un siglo tan dominado por la ciencia como el que nos ha tocado vivir necesita una perspectiva desde la que examinar las creencias científicas que tan a menudo da por supuestas, y la historia es una de las más importantes vías que pueden proporcionárnosla. Si podemos descubrir los orígenes de algunos conceptos científicos modernos y el modo en que han reemplazado a los correspondientes de épocas pretéritas, quizá consigamos valorar de forma inteligente cuáles son sus posibilidades de supervivencia. La presente obra se ocupa básicamente de los conceptos astronómicos, pero existe gran similitud entre estos y los propios de otras ramas del conocimiento científico. En consecuencia, analizando su desarrollo podremos comprender mejor -al menos, así lo esperamos- las teorías científicas en general. (Kuhn 1957, v.i., pp. 3-4; v.c., pp. 26-27)

Por otra parte, Kuhn ha utilizado en sus investigaciones el material de casos confeccionado por Conant y sus colegas, aportando a la misma tarea con su estudio La revolución copernicana. Respecto de este ensayo, el último expresa en el Prólogo:

El profesor Kuhn se enfrenta, no con un caso aislado en la historia de la ciencia, sino con una serie de sucesos influenciados por, y que a su vez influenciaron, la actitud de hombres instruidos cuyos intereses estaban fuera del campo astronómico propiamente dicho. No se ha impuesto la relativamente fácil tarea de relatar la historia del desarrollo de la astronomía durante un período revolucionario. Antes bien, ha conseguido presentar con pleno éxito un análisis de la relación entre teoría, observación y creencia [...]. Este libro no es una relación superficial del trabajo de los científicos. Por el contrario, se trata

\footnotetext{
${ }^{6}$ Sobre ese encuentro entre Kuhn y Conant en 1947 y la significación que tuvo la experiencia en la elaboración de las ideas del primero, véase Kuhn (2000, v.i., pp. 13-32; v.c., pp. 23-45).

${ }^{7}$ Sobre la participación de Leonard Nash y Thomas Kuhn como asistentes en el Programa General de Educación e Historia de la Ciencia en Harvard desarrollado por James B. Conant, puede consultarse Jacobs (2010).
} 
de una completa exposición de una de las fases del trabajo científico y de la que el lector atento podrá extraer interesantes conclusiones sobre la curiosa interacción entre hipótesis y experimento [...] que es la esencia de la ciencia moderna, aspecto ampliamente desconocido por los no científicos. (Kuhn 1957, v.i., pp. xvii-xviii; v.c., p. 20)

Conant y Kuhn sitúan la aproximación histórica a través de los estudios de casos (case studies) como el camino adecuado para la comprensión de la actividad científica, otorgándole a la historia de la ciencia un rol clave para asumir la ciencia como forma fundamental en la educación cultural y así disminuir la brecha entre legos, filósofos, historiadores y científicos (Conant 1947, Kuhn 1957, 1977). La necesidad de clarificar la actividad científica surge del complejo contexto de finalización de la Segunda Guerra y comienzo de la Guerra Fría. La ecuación ciencia-democracia propuesta por John Dewey fue impulsada bajo los auspicios de Robert Merton, Michael Polanyi y el propio James Conant (Mirowsky 2003, pp. 9-18).

\section{La comprensión de la actividad científica a través de esquemas conceptuales}

Una línea de continuidad significativa entre los planteos de Conant y Kuhn es la caracterización que ambos hacen de la dinámica histórica de la ciencia como la sucesión de una unidad de análisis que denominan "esquemas conceptuales". Ninguno de los dos ha ofrecido una definición clara de dichos esquemas, aunque se han servido del concepto utilizándolo para sus interpretaciones de la historia de la ciencia, transformándolo en un concepto operacional para explicitar diferentes aspectos y procesos de la naturaleza de la actividad científica.

Los esquemas conceptuales constituyen un sistema abarcador de diversos componentes relacionados entre sí que articulan el proceso de investigación por el cual los científicos elaboran sus teorías, las ponen a prueba, recolectan nuevos hechos, realizan diversos experimentos y observaciones, evalúan sus resultados y obtienen nuevas aplicaciones. En este sentido, un sistema conceptual se compone de teorías, hechos relacionados con esas teorías, métodos y técnicas, experimentos, observaciones, valoraciones y casos que ilustran determinadas aplicaciones (ejemplares).

Para Conant, la ciencia vista desde un punto de vista dinámico se define como "una interconectada serie de conceptos y esquemas conceptuales que se han desarrollado como un resultado de la experimentación y la observación, capaces de engendrar nueva experimentación y observación" (Conant 1951, v.c., p. 35; también 1965, pp. 12-13). De este modo, sostiene además que "un sistema conceptual cuando es formulado por primera vez puede ser considerado un postulado en gran escala, del cual es posible deducir muchas consecuencias, cada una de las cuales a su vez puede ser el origen de encadenamientos de razonamientos que llevan a deducciones comprobables por experimentación" (Conant 1951 , v.c., p. 58). 
Según Conant, los esquemas conceptuales le otorgan al estudio de la ciencia y su metodología un carácter eminentemente histórico y dinámico en un doble sentido: por un lado, resultan elementos teóricos fructíferos, en tanto dan lugar a una innumerable cantidad de consecuencias observables que pueden ser contrastadas empíricamente, motorizando innovaciones en el terreno científico; por otro, la ciencia explicada como una sucesión de estos adquiere una imagen histórica particular a partir de la consideración de las diversas aproximaciones de dichos esquemas. En tal sentido, la ciencia posee "una condición dinámica cuando se la concibe no como empresa práctica, sino como un proceso para desarrollar esquemas conceptuales. La ciencia adelanta no por acumulación de nuevos hechos [...] sino por el continuo desenvolvimiento de nuevos y fructíferos conceptos" (Conant 1951, v.c., p. 146).

Para Kuhn la ciencia puede ser entendida también como desenvolvimiento y sucesión de nuevos y ricos esquemas conceptuales. Antes de recurrir al término paradigma, en su ensayo La revolución copernicana interpreta el desarrollo histórico de la ciencia en términos de aquellos esquemas conceptuales que utilizan los científicos como guía para sus investigaciones. ${ }^{8} \mathrm{Al}$ respecto, afirma:

El hecho de que el científico utilice tal o cual esquema conceptual en sus explicaciones es un índice de hasta qué punto confía en él, es una muestra de su convicción de que el modelo que emplea es el único válido [...]. La historia de la ciencia está llena de ejemplos de esquemas conceptuales en los que se ha creído de forma fervorosa hasta que fueron reemplazados por otras teorías incompatibles con ellos [...] la adhesión a un determinado esquema conceptual es un fenómeno común en el campo científico [...]. Los esquemas conceptuales son comprensibles y sus consecuencias no se limitan a explicar lo que ya nos es conocido. (Kuhn 1957, v.i., p. 39; v.c., pp. 69-70)

La función cognoscitiva más importante de los esquemas conceptuales consiste en guiar a los científicos a través de un terreno desconocido, indicándoles dónde deben centrar su atención y con qué pueden esperar encontrarse (Kuhn 1957). Esa función cognoscitiva de los esquemas conceptuales está ligada estrechamente con el lenguaje. En ese sentido, en sus trabajos Kuhn ha asociado la noción de "esquema conceptual" a la de una categoría léxica de clasificación o "módulo taxonómico". Al respecto, sostiene:

Pero lo que tengo en mente puede aclararse sugiriendo que podría hablar más propiamente de conceptos que de palabras. Es decir, sería mejor denominar esquema conceptual a lo que he estado llamando una taxonomía léxica, de modo que la "auténtica noción" de un esquema conceptual no es la de un conjunto de creencias, sino la de un modo particular de operar de un módulo mental que es prerrequisito para tener creencias, un modo de operar que proporciona y limita a la vez el conjunto de creencias que es posible concebir. (Kuhn 2000, v.i., p. 94; v.c., p. 118)

En sus trabajos posteriores, principalmente en su ensayo La estructura de las revoluciones científicas, de 1962, Kuhn sustituye la expresión "esquemas concep-

\footnotetext{
${ }^{8}$ Para un análisis sobre el origen de la expresión "esquema conceptual" en el pensamiento de Kuhn y de la aplicación que hace del término en el estudio de la Revolución Copernicana, véase Swerdlow (2004).
} 
tuales" por el término "paradigma” y especifica su función cognoscitiva. En tal sentido, la idea de "esquema conceptual" ha sido fundamental en Kuhn para plantear el problema de la inconmensurabilidad y las relaciones entre un paradigma y otro en términos de traducción e interpretación. Puede considerarse una de las dimensiones de su pensamiento que expresan originalidad respecto de las ideas de Conant, pero también uno de los aspectos fuertemente atacados por sus críticos.

La noción de "esquema conceptual", entendido como elemento organizador de los datos de la experiencia, ha sido tomada en común por Conant y Kuhn de los trabajos del lógico Willard Quine (1908-2000). Los primeros asumen el principio sugerido por este último, de considerar como similares la aceptación de una ontología y la aceptación de una teoría científica, una situación en la cual "adoptamos, al menos hasta el punto en que es razonable, el simple esquema conceptual dentro del cual los fragmentos desordenados de cruda experiencia pueden ser ajustados y organizados" (Quine 1963, p. 16).9

Asimismo, la idea de "esquema conceptual" ha sido prolífica en la filosofía del lenguaje y de la ciencia contemporánea, dando lugar a significativas discusiones que han enfrentado realismo, empirismo, criticismo y relativismo, entre otras posiciones filosóficas. ${ }^{10} \mathrm{El}$ mismo Kuhn ha intervenido en algún aspecto de estas discusiones al plantear el problema de la inconmensurabilidad y los procedimientos lingüísticos implicados en ella (Kuhn 1970, v.i., pp. 198-204; v.c., pp. 336-345; y 2000, v.i., pp. 33-57; v.c., pp. 47-75). ${ }^{11}$

Para ambos historiadores, los esquemas conceptuales o paradigmas (Kuhn) no se rechazan sin más por uno o varios ejemplos en contrario, por algunos hechos que no pueden ser adaptados al esquema explicativo. Pero tampoco aquellos se rechazan sin ser sustituidos por otros que tomen su lugar. Sobre esto Conant afirma:

en cuestiones complejas de la ciencia, de lo que se trata es de explicar una diversidad de hechos y coordinarlos en un plan conceptual; un solo hecho no basta para demoler el esquema anterior [...] jamás se descarta un esquema conceptual solo porque unos cuantos hechos obstinados no encajen en el esquema; se modifica o reemplaza un concepto por otro mejor, pero nunca se abandona sin que otro ocupe su lugar. (Conant 1947, v.c., p. 94) ${ }^{12}$

\footnotetext{
${ }^{9}$ Traducción mía. Otras referencias importantes sobre la influencia de Quine en el pensamiento de ambos historiadores de la ciencia son Conant (1965, pp. 13-14), Kuhn (1970, pp. vi, 201 y ss.; v.c., pp. 47, 340 y ss).

${ }^{10}$ En gran medida ello ha sido por los trabajos de Willard Quine y Donald Davidson. Véase, por ejemplo, Davidson (1974).

${ }^{11}$ Sobre el problema de la inconmensurabilidad y los esquemas conceptuales resultan interesantes las comparaciones que se han realizado entre la noción de paradigma en Kuhn y lo que Ludwik Fleck llamó "estilo de pensamiento" (Denkstil). Al respecto, véase Babich (2003).

${ }^{12}$ En otra parte sostiene: "Podemos dejar sentado como uno de los principios aprendidos en la historia de la ciencia el de que una teoría es siempre suplantada por otra teoría mejor, nunca por hechos contradictorios. $\mathrm{Al}$ principio se procuró que armonizasen los hechos contradictorios con el esquema conceptual existente, mediante una modificación del concepto. Solo de la combinación del concepto nuevo con hechos contradictorios a las ideas antiguas resulta al fin una revolución científica. Y una vez que esta se ha producido, entonces siguen a los pocos años los descubrimientos, y la correspondiente rama de la ciencia en cuestión progresa a saltos" (Conant 1947, v.c., pp. 50-51). La afirmación sobre el progreso científico a través de "saltos",
} 
Por su parte, Kuhn se refiere al cambio de paradigma en el mismo sentido, cuando expresa que "una teoría científica solo se considerará inválida si hay disponible un candidato alternativo para ocupar su lugar" y agrega más adelante que "rechazar un paradigma sin sustituirlo a la vez por otro es rechazar la propia ciencia” (Kuhn 1970, v.i., pp. 77 y 79; v.c., pp. 165 y 168).

De esta manera, la dinámica histórica de la ciencia queda expresada en la sucesión de esquemas conceptuales o paradigmas que se reemplazan unos a otros, cuando el precedente no explica hechos contradictorios o anómalos sustanciales y el siguiente está formado para poder explicar las inconsecuencias que se han hecho evidentes.

Si bien ambos historiadores comparten la comprensión de la actividad científica a través de esquemas conceptuales, Conant sostiene la idea de un desarrollo histórico de la ciencia donde predomina un enfoque acumulativo, característica que distingue esta forma de conocimiento de otras actividades sociales y culturales como el arte o la filosofía (Conant 1947, v.c., pp. 35-36). Para Kuhn, por su parte, el reconocimiento de momentos de discontinuidad y de un tipo de progreso por medio de cambios revolucionarios -cuando un esquema conceptual o paradigma sustituye a otro- resulta una perspectiva central en su pensamiento que lo diferencia de las ideas de aquel. ${ }^{13}$

\section{Práctica científica y desarrollo psicogenético del niño}

Otro punto de continuidad entre el pensamiento de Conant y el de Kuhn se expresa en la comparación que ambos hacen entre el trabajo del científico en el proceso de investigación y la génesis de las ideas en el niño, a partir de los aportes de los trabajos de Jean Piaget (1896-1980).

Conant asimila el trabajo que realizan los científicos para articular sus esquemas conceptuales con la experiencia al aprendizaje que efectúan los niños en los primeros años para diferenciar y agrupar los objetos en clases. Esta idea aparece como una fuerte sugerencia en el pensamiento de Conant, útil para profundizar el proceso histórico de elaboración del conocimiento científico. Sobre este aspecto afirma: "Pero más fundamental [...] es la conexión entre los intentos de los científicos que generación tras generación procuraron desarrollar y mejorar una serie de esquemas relativos a la experimentación y el proceso mediante el cual un niño llega a distinguir los objetos y las personas que le rodean en su primera etapa de vida" (Conant 1951, v.c., p. 41).

La conexión que sugiere Conant entre el trabajo del científico y el aprendizaje de los niños surge del enfoque de aproximación histórica a la ciencia que comparte con Kuhn. En tal sentido, la idea sugerida por el primero es retoma-

con excepción de lo que pueda especificarse como "mejor", van en el sentido de lo que plantea Kuhn de manera más decidida como un progreso por medio de "revoluciones científicas" (Kuhn 1970, v.i., pp. 160. 173; v.c., pp. 281-300).

${ }^{13}$ Esta diferencia entre Conant y Kuhn ha sido señalada por Hershberg (1993, p. 410). 
da posteriormente por el segundo en sus consideraciones sobre el problema de la inconmensurabilidad y la valoración de teorías por parte de las comunidades científicas. Este centra su atención fundamentalmente en las investigaciones desarrolladas por Piaget y su escuela sobre los procesos psicogenéticos del aprendizaje de los niños. La participación de Kuhn en las reuniones y debates del Centro Internacional de Epistemología Genética fundado por Piaget (Piaget 1970, v.c., pp. 25-26), así como las referencias del historiador de la ciencia a los trabajos del psicólogo ginebrino, revelan su interés por esta idea.

La sugerencia inicial de Conant se convierte luego para los planteos de Kuhn en un modo fundamental de preguntar a los científicos a través de la historia, lo que constituirá otro aspecto de originalidad respecto de su maestro. Una primera referencia de Kuhn a las investigaciones de Piaget es en relación con el pensamiento animista de los niños y las sociedades primitivas sobre el mundo, en comparación con la concepción aristotélica del espacio y el tiempo (Kuhn 1957, v.i., pp. 97-99; v.c., pp. 139-142). Posteriormente, durante los años en los cuales elabora las ideas de su ensayo fundamental, sostiene que:

pasé la mayor parte del tiempo explorando campos que aparentemente carecían de relación alguna con la historia de la ciencia, aunque ahora la investigación descubre en ellos problemas semejantes a los que la historia ponía ante mis ojos. Una nota hallada por casualidad me llevó hasta los experimentos mediante los cuales Jean Piaget ha iluminado no solo los diferentes mundos del desarrollo infantil, sino también el proceso de transición de cada uno de ellos al siguiente. (Kuhn 1970, v.i., p. xi; v.c., pp. 47) ${ }^{14}$

Las investigaciones piagetianas constituyen una base para contrastar y evaluar el desarrollo histórico de enfoques antiguos. Al respecto, Kuhn afirma que "temas como las concepciones que los niños tienen del espacio, del tiempo, el movimiento o el propio mundo han revelado repetidas veces sorprendentes paralelos con las concepciones que de estos mismos asuntos han tenido los científicos adultos de la antigüedad" (Kuhn 1977, v.i., p. 21; v.c., p. 46). La incidencia del pensamiento de Piaget se revela decisiva en Kuhn, lo cual es reconocido por este cuando expresa:

Hace casi veinte años que descubrí, y más o menos al mismo tiempo, tanto el interés intelectual por la historia de la ciencia, como los estudios psicológicos de Jean Piaget. Desde entonces, ambas inquietudes han influido recíprocamente tanto en mi mente como en mi trabajo. Parte de lo que sé sobre la forma de interrogar a los científicos que ya han muerto lo aprendí examinando la forma en que Piaget interroga a los niños que estudia [...]. Incluso en terrenos como los de la causalidad, acerca de la cual quizá no se esté completamente de acuerdo, me siento orgulloso de reconocer las huellas indelebles de la influencia de Piaget. (Kuhn 1977, v.i., pp. 21-22; v.c., p. 46)

Las ideas de Piaget le ofrecen a Kuhn un modo de interrogar a los científicos en la historia, pero al mismo tiempo constituyen una clave para entender el pro-

\footnotetext{
${ }^{14}$ Sobre esa referencia al psicólogo ginebrino, Kuhn comenta: "Una nota al pie de página en el libro de R. K. Merton Ciencia, sociedad y tecnología en el siglo diecisiete en Inglaterra me condujo a la obra del psicólogo evolucionista Jean Piaget" (Prólogo a Fleck 1979, v.i., p. viii; v.c., p. 115).
} 
ceso por el cual las comunidades científicas asimilan y aprenden sus esquemas conceptuales o paradigmas. Evidenciando la línea de continuidad con Conant, Kuhn sitúa el aprendizaje ostensivo mediante el cual un niño divide y agrupa en clases los objetos, en el fondo de la cuestión sobre la adquisición de ejemplares paradigmáticos de una comunidad científica por parte de jóvenes investigadores, así como las consecuencias de la inconmensurabilidad para la práctica de la ciencia normal (Kuhn 1970, v.i., pp. 187-204; v.c., pp. 321-345; 1977, v.i., pp. 293-319; v.c., pp. 317-343).

\section{Los factores institucionales de la ciencia}

La consideración de los factores sociales de la ciencia, principalmente los institucionales, expresan otra línea de continuidad entre Conant y Kuhn. Para el primero: "Los siglos XVII y XVIII fueron la época de las sociedades de eruditos, en especial la Royal Society londinense y la Académie des Sciences de Francia" (Conant 1951, v.c., pp. 27-28). En tal sentido, sostiene que el rol de las sociedades científicas en el fomento de la nueva filosofía y el patrocinio de la publicación de libros y revistas requieren especial atención, debido a que ellas adquirieron una gran importancia en el crecimiento del sentimiento profesional acerca de la ciencia, cambiando la atmósfera paulatinamente (Conant 1951, v.c., pp. 19 y 74; 1947, v.c., p. 23). Estas instituciones transformaron la ciencia en una actividad social organizada, mostrando que:

los investigadores primitivos de fenómenos físicos, químicos y biológicos trabajaron casi siempre aisladamente, y cómo a mediados del siglo diecisiete, en diferentes países, estos aficionados se unieron, creando las sociedades científicas. Se estudiaría el papel de estas sociedades en los años subsiguientes, dedicando atención especial al incremento de bibliografía científica y a la gradual evolución de la investigación científica como una ocupación profesional. [...] Tómese, por ejemplo, el papel de las universidades. Me inclino a pensar que sería muy provechoso el estudio de la historia de estas instituciones desde el siglo décimosexto al vigésimo.

El hecho de que el progreso de la ciencia pura, con escasas excepciones, se haya debido poco a la obra de los universitarios de mediados del siglo XVII a mediados del XIX no resta importancia a la historia. Esto requirió que se creasen otras varias instituciones para el fomento de la ciencia y, por supuesto, aumentó considerablemente la importancia de las sociedades científicas. (Conant 1947, v.c., p. 115)

El estudio de las sociedades científicas relacionado con el naciente sentimiento profesional de la actividad, así como el de las universidades vinculado a la historia religiosa y a los debates cosmológicos y epistemológicos de los centros del saber, tiene utilidad por cuanto relaciona la evolución de la ciencia con el clima de opinión de cada época (Conant 1947, v.c., pp. 115-116).

Por su parte, Kuhn sostiene que el análisis de las instituciones científicas constituye una de las formas más antiguas en el intento de situar la ciencia en un contexto cultural. Los materiales sobre las instituciones científicas -la ma- 
yoría sobre el siglo XVII- son útiles como fuentes para el historiador interesado en examinar este y otro tipo de instituciones como las educativas, que pueden promover o inhibir el avance de la ciencia (Kuhn 1977, v.i., p. 113; v.c., p. 137). De tal forma, siguiendo la propuesta de Conant, Kuhn señala la importancia de analizar las transformaciones y la continuidad del factor institucional en el estudio de las revoluciones científicas. Sobre esto afirma:

\begin{abstract}
El desarrollo técnico interno no fue [...] el único requisito para la aparición de una ciencia con significación social [...]. Durante el siglo XIX, la estructura institucional y social de las ciencias se transforma de maneras no anunciadas por la Revolución científica. Empezando en 1780 y continuando a través de la primera mitad del siglo siguiente, las entonces recién formadas sociedades de especialistas en las distintas ramas de la ciencia tomaron la primacía que las sociedades nacionales, que abarcaban toda la ciencia, no habían logrado asumir plenamente. Al mismo tiempo, proliferaron las revistas científicas privadas, en especial las referentes a disciplinas específicas, y poco a poco fueron reemplazando a las publicaciones de las academias nacionales, anteriormente el medio casi exclusivo de comunicación entre científicos. Un cambio similar se aprecia en la educación científica y en el lugar que se le da a la investigación [...] esas formas institucionales nuevas son el fundamento de una segunda revolución científica que puede ubicarse en la primera mitad del siglo XIX, y es un acontecimiento histórico de por lo menos tanta importancia para el entendimiento de los tiempos modernos como la primera. (Kuhn 1977, v.i., pp. 146-147; v.c., pp. 170-171)
\end{abstract}

De este modo, para ambos historiadores el análisis institucional y social de la actividad científica constituye una preocupación compartida tan importante como el abordaje de la Revolución Científica, en el intento por comprender el desarrollo de la ciencia en la historia. En tal sentido, el estudio de las instituciones científicas y educativas revela la conexión entre las ideas y la cultura de una época con la práctica científica, al tiempo que expresan las formas de organización social que adquiere esa actividad.

\title{
6. La dialéctica entre lo empírico y lo teórico
}

Una particular y común concepción de las vinculaciones entre lo empírico (observaciones y experimentación) y lo teórico (esquemas conceptuales o paradigmas) aparece en las propuestas de ambos. Esta concepción la expresa Conant en su descripción de la naturaleza de la actividad científica, cuando sostiene que "como aproximación primera, podemos decir que la ciencia brota de las otras actividades progresistas del hombre en el grado en que de los experimentos y observaciones surgen nuevos conceptos, y que los nuevos conceptos conducen a su vez a experimentos y observaciones ulteriores" (Conant 1947, v.c., p. 39). La dialéctica entre el nivel fáctico y el nivel teórico constituye para él la cualidad dinámica de la ciencia, expresada como evolución de planos conceptuales en un movimiento de aproximaciones sucesivas, y que solo es accesible mediante un enfoque histórico (método de casos). 
Por otro lado, esta dialéctica es elevada a un principio fundamental que sostiene Conant acerca del desarrollo histórico de la ciencia: de experimentos u observaciones surgen nuevos conceptos que a su vez determinan nuevos experimentos u observaciones (Conant 1947, v.c., pp. 109-112). Un objetivo propuesto por él en uno de sus principales trabajos es hacer evidente este principio cuando afirma:

Confío en poder ilustrar la complicada relación entre el experimento o la observación y el desarrollo de nuevos conceptos y nuevas generalizaciones; en resumen, la forma en que los conceptos nuevos van saliendo de los experimentos, cómo un plan conceptual resulta adecuado durante un tiempo y luego es modificado o desplazado por otro. (Conant 1947, v.c., p. 33)

Las novedades fácticas y las novedades teóricas constituyen, según Kuhn, dos fuentes fundamentales de innovación científica (Kuhn 1970, v.i., pp. 52-76; v.c., pp. 129-164). Las primeras se vinculan directamente con la percepción de las anomalías y con la emergencia histórica de descubrimientos de nuevos hechos científicos; las segundas provienen de las crisis teóricas, de la necesidad de ajuste y de la proliferación de nuevos esquemas conceptuales que expliquen las anomalías y den respuesta a las crisis. Kuhn reconoce una vinculación intrínseca y una dinámica interdependiente entre novedades fácticas y teóricas cuando sostiene:

El descubrimiento comienza tomando conciencia de una anomalía, es decir, reconociendo que la naturaleza ha violado de algún modo las expectativas, inducidas por el paradigma que gobierna la ciencia normal. Prosigue luego con una exploración más o menos amplia del área de la anomalía, y se cierra solo cuando la teoría paradigmática se ha ajustado para que lo anómalo se vuelva algo esperado. La asimilación de un nuevo tipo de hecho exige un ajuste de la teoría que no se limita a ser un añadido, y hasta que no se termina dicho ajuste $[\ldots]$ el hecho nuevo no es en absoluto un hecho plenamente científico. (Kuhn 1970, v.i., pp. 52-53; v.c., p. 130)

En el mismo sentido, en el texto citado expresa más adelante que:

descubrir un nuevo tipo de fenómeno es necesariamente un suceso complejo que entraña reconocer tanto que algo es, como qué es [...]. Pero si tanto la observación como la conceptualización, el hecho y la asimilación a una teoría, se encuentran inseparablemente unidos en el descubrimiento, entonces el descubrimiento es un proceso que ha de llevar tiempo [...] el descubrimiento entraña un proceso extenso, aunque no necesariamente largo, de asimilación conceptual. (Kuhn 1970, v.i., pp. 55-56; v.c., p. 134)

Esta dialéctica entre hechos y esquemas conceptuales, señalada tanto por Conant como por Kuhn, hace difusa una clara delimitación entre descubrimiento e invención. El primero afirma que en el estudio histórico de la ciencia, "la diferencia entre invención y descubrimiento científico puede parecer pequeña en unos pocos casos" (Conant 1947, v.c., p. 37). En el mismo sentido, Kuhn señala que "tal distinción entre descubrimiento e invento o entre hecho y teoría, pronto resultará ser extremadamente artificial" (Kuhn 1970, v.i., p. 52; v.c., p. 130). 
Para ellos, la particular relación entre lo teórico y lo empírico que revela el análisis histórico de la ciencia promueve la reconsideración no solo de la distinción clásica entre descubrimiento e invención, sino también del rol que juega el factor técnico en el desarrollo del conocimiento científico.

\section{El factor técnico en el desarrollo histórico de la ciencia}

La perspectiva de valoración del factor técnico en la historia de la ciencia constituye otro punto de continuidad entre ambos historiadores. Conant y Kuhn señalan dos aspectos que no deben confundirse: la ciencia y sus aplicaciones técnicas (Conant 1947, Kuhn 1977, v.i., pp. 141-147; v.c., pp. 165-170). Esta distinción surge del intento por comprender la ciencia como actividad social organizada y su influencia sobre la sociedad a través de los desarrollos técnicos.

Conant destaca el factor técnico como un principio de la comprensión histórica del desarrollo del conocimiento científico. Sostiene que su importancia radica en que los nuevos desarrollos técnicos que resultan de la experimentación pueden incentivar nuevos experimentos. Por ello, las técnicas de experimentación pueden caracterizarse como elementos altamente revolucionarios debido a sus posibilidades de innovación y de conducir a nuevos descubrimientos (Conant 1947, v.c., pp. 113-114).

De manera similar, Kuhn afirma que una de las causas de la gran transformación y el fuerte desarrollo de la ciencia a partir de 1870 es precisamente la incidencia del rol profundamente revolucionario del factor técnico. Aspecto clave en el estudio histórico de la ciencia para el que, según expresa, "pocos historiadores socioeconómicos cuentan con las herramientas para seguir los aspectos técnicos de los avances que repentinamente hacen que una ciencia comience a producir materiales nuevos e inventos" (Kuhn 1977, v.i., p. 146; v.c., p. 170).

Ambos historiadores reconocen la importancia del factor técnico en la posibilidad de ampliar el conjunto de hechos que un determinado esquema conceptual puede explicar en contraposición a otro. Para ellos, el elemento técnico junto a la ampliación de la estructura institucional de la ciencia constituyen dos causas que explican la aparición de una ciencia con significación social durante el siglo XIX.

\section{Crítica a los libros de texto para la formación de estudiantes e investigadores}

La última línea de continuidad que se señala en este trabajo entre los planteos de Conant y Kuhn es la preocupación común respecto de la imagen de la ciencia que ofrecen los libros de texto con los que se forman los jóvenes investigadores. 
Conant presenta una crítica a ciertos conceptos que aparecen en libros de texto sobre la investigación científica. Respecto de las nociones de "teoría" e "hipótesis" afirma:

El lector puede recordar que la palabra teoría e hipótesis se emplean frecuentemente para describir los esquemas y los modelos o ilustraciones que "explican" tales esquemas. Una exposición de la definición de teoría e hipótesis se da con frecuencia en textos elementales, pero yo creo que tal exposición tiene valor dudoso. (Conant 1947, v.c., p. 61)

Para Kuhn, este cuestionamiento a los conceptos sobre la ciencia expuestos en los libros de texto se convierte en una preocupación central que atraviesa todo el capítulo XI de su ensayo de 1962. Allí analiza la imagen de la ciencia que ofrecen dichos textos en relación con la invisibilidad de las revoluciones científicas y el modo en que ellos ocultan los cambios de paradigma. Sobre este aspecto expresa que:

Tanto los científicos como los legos toman en gran parte la imagen que tienen de la actividad científica creadora de una fuente autorizada que oculta sistemáticamente la existencia y el significado de las revoluciones científicas. [...] Como fuente de esta autoridad, pienso principalmente en los libros de texto científicos junto con las divulgaciones y las obras filosóficas que los toman como modelo. (Kuhn 1970, v.i., p. 136; v.c., pp. 247-248)

Los libros de texto contienen poca historia de la disciplina de que tratan (generalmente en un capítulo introductorio o en referencias dispersas). Al mismo tiempo, la imagen que ellos brindan de la ciencia es acumulativa y lineal en relación con la situación actual y con el estado presente de la disciplina en cuestión. De este modo, los libros de texto truncan el sentido histórico de una disciplina, haciendo invisibles los cambios (revoluciones científicas) que dieron lugar o sentaron las bases del campo donde se desarrolla una determinada actividad científica. Esta deformación de la historia de una disciplina en el marco de una tradición de investigación tiene un rol pedagógico importante.

En este sentido, la función principal de los libros de texto científicos consiste en su potencialidad para lograr una efectiva educación de los estudiantes e investigadores en el contexto de una tradición científica, por medio de las aplicaciones o ejemplares que ofrece el paradigma. Al respecto, Kuhn sostiene:

Las investigaciones históricas minuciosas de una especialidad dada en un momento determinado muestran un conjunto de ilustraciones recurrentes y casi estándares de diversas teorías en sus aplicaciones conceptuales, observacionales e instrumentales. Se trata de los paradigmas de la comunidad puestos de manifiesto en los libros de texto, en las clases y en las prácticas de laboratorio. Los miembros de la comunidad correspondiente aprenden el oficio estudiándolos y practicando con ellos. (Kuhn 1970, v.i., p. 43; v.c., p. 117)

$\mathrm{Y}$ agrega que

Una teoría novedosa se anuncia siempre junto con sus aplicaciones a algún abanico concreto de fenómenos naturales, sin los cuales ni siquiera podría ser candidata a la aceptación. Una vez que ha sido aceptada, estas mismas aplicaciones u otras distintas acompañarán a la teoría en los textos en los 
que los futuros practicantes aprenderán su oficio. (Kuhn 1970, v.i., p. 46; v.c., p. 122$)^{15}$

Esta crítica compartida por Conant y Kuhn a los libros elementales utilizados para la enseñanza de la ciencia ha llevado a distinguir, además de los clásicos contextos de descubrimiento (innovación), justificación (valoración) y aplicación de la ciencia, un componente constitutivo esencial y tan importante como los anteriores: su contexto educativo (Echeverría 1995).

\section{Consideraciones finales}

El recorrido hecho hasta aquí pretende ser un aporte comparativo a la comprensión de los planteos de James B. Conant y Thomas S. Kuhn, exponentes claves de la filosofía y la historia de la ciencia contemporánea.

Los aspectos analizados del pensamiento de ambos sobre el desarrollo histórico de la actividad científica resultan relevantes para entender la incidencia que el enfoque propuesto por Conant tuvo en la génesis de las ideas y del pensamiento de Kuhn.

Las analogías presentes en los puntos de comparación considerados revelan las líneas de continuidad que existen entre ambos historiadores en el marco de un mismo ambiente intelectual. En tal sentido, Kuhn se sitúa como parte de la tradición de Harvard a la que pertenecen George Sarton (1884-1956), Leonard Nash o I. B. Cohen (1914-2003), cuyos aportes a la consideración histórica de la ciencia han sido en cierta forma predominantes.

Con todo, existen características del pensamiento de Kuhn que lo diferencian del de su mentor y le confieren originalidad. La construcción de una mirada sociológica, a la que contribuyó la lectura de Ludwik Fleck, así como la elaboración de una perspectiva discontinuista de la historia de la ciencia aportada por los trabajos de Alexandre Koyré constituyen teorizaciones que Kuhn incorporó y modeló de manera personal (Solís 2007). Al mismo tiempo, son características fundamentales de su pensamiento que contrastan con el enfoque acumulativo del progreso científico presente en Conant. La primera profundizó y reorientó sus investigaciones histórico-epistemológicas hacia los compromisos, valores, métodos y consensos expresados en el componente comunitario de la actividad científica. La segunda tiene correspondencia en sus planteos sobre la inconmensurabilidad y el holismo de los paradigmas, como también en su concepción sobre el progreso a través de revoluciones. Estas dos vertientes resultan capitales en la génesis del pensamiento y en el armazón teórico de quien revolucionó los modos de concebir la producción y el desarrollo científico.

\footnotetext{
${ }^{15}$ En otra parte, tomando la terminología del programa de reconstrucción estructural de las teorías empíricas, Kuhn expresa: "En gran medida, la educación de un científico, o al menos de un físico, consiste en enseñar al estudiante a hacer la transición desde los modelos parciales potenciales a los modelos parciales. Para eso están los laboratorios estudiantiles y los problemas al final de los capítulos de los manuales” (Kuhn 2000, v.i., p. 178; v.c., pp. 213-214).
} 
Por otra parte, se han señalado dos diferencias relevantes entre ambos respecto de la compresión del componente comunitario y la dinámica interna de la ciencia. Primeramente, en Kuhn no aparecen las referencias que hace Conant sobre la actividad científica como salvaguardia de la democracia contra el comunismo en el contexto de la Guerra Fría. Las expresiones políticas resultan en Kuhn metáforas empleadas para caracterizar el trabajo interno de las comunidades científicas. Además, la cerrada autonomía derivada del paradigma con la que entiende a esas últimas es un rasgo criticado por Conant, para quien los esquemas conceptuales son más permeables al ensayo y error y a los procesos de racionalidad comunicativa que posibilitan los acuerdos (Fuller 2001, pp. 180-181).

En lo relativo a las dimensiones analizadas en este artículo, cada una de ellas se incluye en los planteos de Conant y todas se revelan comunes a preocupaciones presentes también en el pensamiento de Kuhn, constituyéndose en problemas fundamentales en los enfoques propuestos por ambos para la comprensión del desarrollo histórico de la ciencia. Asimismo, consideradas en conjunto, esas dimensiones constituyen tópicos centrales en la discusión filosófica e histórica sobre la ciencia durante la segunda mitad del siglo XX.

De esta manera, se evidencia la significación que las líneas de continuidad presentadas tienen para los debates contemporáneos y, al mismo tiempo, para las posibilidades de comprensión entre las propuestas elaboradas por Conant y Kuhn y otras perspectivas actuales como el constructivismo, el cambio conceptual o la concepción estructural de las teorías. 


\section{Bibliografía}

Babich, B. E. (2003), "From Fleck's Denkstil to Kuhn's Paradigm: Conceptual Schemes and Incommensurability", International Studies in the Philosophy of Science 17(1): 75-92.

Conant, J. B. (1947), On Understanding Science. An Historical Approach, New Haven: Yale University Press. (Versión castellana: La comprensión de la ciencia, Buenos Aires: Plaza \& Janés, 1963.)

Conant, J. B. (1951), Science and Common Sense, New Haven: Yale University Press. (Versión castellana: La ciencia y el sentido común, Buenos Aires: Kraft, 1953.)

Conant, J. B. (ed.) (1957a), Harvard Case Histories in Experimental Science, Cambridge, MA: Harvard University Press.

Conant, J. B. (1957b), "Forward", en Kuhn (1957), pp. xiii-xviii. (Versión castellana: "Prólogo", en Kuhn (1978), pp. 15-21.)

Conant, J. B. (1965), Two Modes of Thought. My Encounters with Science and Education, New York: Pocket Books.

Davidson, D. (1974), "The Very Idea of a Conceptual Scheme”, Proceedings and Addresses of the American Philosophical Association 47: 5-20.

Echeverría, J. (1995), Filosofía de la ciencia, Madrid: Akal.

Fleck, L. (1979), Genesis and Development of a Scientific Fact, Chicago: The University of Chicago Press. (Versión castellana del prólogo de Kuhn: Metatheoria 1(1) (2010): 115-118.)

Fuller, S. (2001), Thomas Kuhn. A Philosophical History for our times, Chicago: The University of Chicago Press.

Hershberg, J. G. (1993), James B. Conant: Harvard to Hiroshima and the Making of the Nuclear Age, New York: Alfred A. Knopf.

Jacobs, S. (2010), “J. B. Conant's Assistant: Science as Depicted by Leonard K. Nash, Including Reference to Thomas Kuhn", Perspective on Science 18(3): 328-351.

Kuhn, T. S. (1957), The Copernican Revolution, Cambridge, MA: Harvard University Press. (Versión castellana: La revolución copernicana, Barcelona: Ariel, 1978.)

Kuhn, T. S. (1970), The Structure of Scientific Revolutions, $2^{\text {nd }}$ enlarged ed., Chicago: University of Chicago Press. (Versión castellana: La estructura de las revoluciones científicas, México: Fondo de Cultura Económica, 2007).

Kuhn, T. S. (1977), The Essential Tension: Selected Studies in Scientific Tradition and Change, Chicago: University of Chicago Press. (Versión castellana: La tensión esencial. Estudios selectos sobre la tradición y el cambio en el ámbito de la ciencia, México: Fondo de Cultura Económica, 1996.)

Kuhn, T. S. (2000), The Road Since Structure: Philosophical Essays, 1970-1993, with an Autobiographical Interview, Chicago: University of Chicago Press. (Versión castellana: El camino desde la estructura: Ensayos filosóficos, 1970-1993, con una entrevista autobiográfica, Barcelona: Paidós, 2002).

Mirowsky, P. (2003), "The Scientific Dimensions of Social Knowledge and their Distant Echoes in $20^{\text {th }}$ Century American Philosophy of Science", University of Notre Dame, http://www.newschool.edu/scepa/events/papers/workshop/mirowski_090303.pdf (acceso 20 de febrero, 2011). 
Pacheco, P. (2005), Origen, incorporación y función de los estudios de casos como metodología de la historia de las ciencias, Tesis de Licenciatura, Facultad de Filosofía y Letras, Universidad Nacional de Cuyo.

Piaget, J. (1970), Psychologie et épistémologie, Paris: Denoël. (Versión castellana: Psicología y epistemología, Buenos Aires: Emecé, 1983.)

Quine, W. V. O. (1963), From a Logical Point of View, $2^{\text {nd }}$ revised ed., New York: Harper \& Row.

Solís, C. (2007), “Una revolución del siglo XX”, en Kuhn (2007), pp. 9-43.

Swerdlow, N. M. (2004), “An Essay on Thomas Kuhn's First Scientific Revolution, The Copernican Revolution", Proceedings of the American Philosophical Society 148(1): 64-120. 